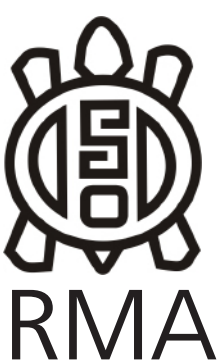

Dossier

\title{
Identificación interespecífica de camélidos en el valle de Ambato (Catamarca, Argentina): una aproximación a la problemática desde distintas líneas de análisis
}

Mariana Dantas*

*CONICET, Museo de Antropología, Facultad de Filosofía y Humanidades, Universidad Nacional de Córdoba, Argentina.

E-mail: dantasmariana@hotmail.com

\begin{abstract}
Resumen
La identificación interespecifica de camélidos y la distinción entre morfotipos de llamas en los materiales óseos recuperados en sitios arqueológicos constituyen dos aspectos de suma importancia dentro de los estudios zooarqueológicos del noroeste argentino y de la región andina en general. En este trabajo se pretende contribuir a esta problemática, mediante el entrecruzamiento de distintas líneas de análisis aplicadas sobre los restos de camélidos procedentes del Valle de Ambato, Catamarca, entre los siglos VI y X d.C. De este modo, la integración de estudios osteométricos y de isótopos estables posibilitó establecer la existencia en este valle de camélidos domésticos en función de su tamaño relativo y una dieta selectiva concentrada en plantas $C_{4}$. Dentro de aquellos también fue posible distinguir dos grupos que podrían responder a distintas variedades de tamaños: un grupo de animales de mayor porte, o llamas grandes, y otro de menor porte, ollamas pequeñas, cuyo tamaño se superpone con el rango de tamaños de los guanacos norandinos. En definitiva, se puede afirmar, que la combinación de ambos análisis permitió alcanzar un grado de diferenciación que de otro modo no hubiera sido posible.
\end{abstract}

Palabras claves: Zooarqueología; Camélidos Sudamericanos; osteometría; isótopos estables de carbono; Aguada de Ambato.

Interspecific identification of camelids in Ambato valley (Catamarca, Argentina): an approach to the issue from different analytical frameworks

\begin{abstract}
Interspecific identification of camelids and distinction between llamas' morphotypes in bone materials from archaeological sites are two major aspects in zooarchaeological studies of northwestern Argentina and the Andean region in general. This paper aims at contributing to the issue along different analytical approaches applied to camelids remains from Ambato Valley, Catamarca, between the 6th and 10th centuries AD. Thus, the integration of osteometric and stable isotope studies allowed establishing/confirming the presence of domestic camelids in this valley, based on their relative size and a selective diet mostly derived from C4 plants. It was also possible to distinguish two probably differently-sized groups of animals: one of larger animals or large llamas, and one of smaller size or small llamas, whose dimensions overlap with those of North Andean guanacos. A combination of both analyses allowed reaching a degree of differentiation not possible otherwise
\end{abstract}

Keywords: Zooarchaeology; South American Camelids; osteometry; carbon stable isotopes; Aguada Culture.

Dentro de los estudios zooarqueológicos del noroeste argentino y de la región andina en general, la identificación interespecífica de camélidos y la distinción entre morfotipos de llamas en los materiales óseos recuperados en sitios arqueológicos son dos temas de gran importancia y ampliamente investigados (Cardich e Izeta 1999-2000, Cartajena Fasting 2009, Elkin 1996, Izeta 2004, 2007a, Izeta et al. 2009b, Kent 1982, Mengoni Goñalons y Yacobaccio 2006, Olivera y Elkin 1994, Srur e Izeta 2008, Yacobaccio 2010, Yacobaccio et al. 1997/1998, entre otros). En Sudamérica, la familia
Camelidae está compuesta actualmente por cuatro especies, dos silvestres, Lama guanicoe (guanaco) y Vicugna vicugna (vicuña), y dos domesticadas, Lama glama (llama) y Lama (Vicugna) pacos (alpaca). En los Andes Centro Sur tienen un gradiente de tamaño corporal que comienza por la llama, que es la de mayor tamaño, posteriormente se encuentran el guanaco, la alpaca y, por último, la vicuña, que es la más pequeña (Mengoni Goñalons 2008, Mengoni Goñalons y Yacobaccio 2006, Olivera y Elkin 1994). Los guanacos son los que tienen más amplia distribución, encontrándose por toda el 
área andina sudamericana y la patagonia Argentina, desde el nivel del mar hasta los 4.857 msnm. En tanto, la presencia de las vicuñas se limita a los ecosistemas puneños y altoandinos, por arriba de los 3.300 msnm, llegando en Argentina hasta alrededor de los 3.000 m (Laker et al. 2006, Wheeler 1995, 2006). Entre las especies domésticas, las llamas son las más adaptables, ya que se ha registrado su presencia en ambientes de altura, valles y zonas costeras, teniendo una amplia distribución geográfica, desde Ecuador hasta el norte y centro de Chile y el noroeste de Argentina (Mengoni Goñalons 2008, Wheeler et al. 1995). Comparativamente, las alpacas tienen una distribución más restringida, circunscribiéndose a la cuenca del Titicaca, la puna del norte de Chile y Perú central y las zonas costeras del norte y sur de Perú (Mengoni Goñalons 2008, Wheeler 1995).

La diferenciación entre estas cuatro especies es sumamente compleja y se basa en varios indicadores, dentro de los cuales, los mayormente utilizados son la morfología dentaria (Wheeler 1982), el método osteométrico (Elkin 1996, Izeta 2004, 2007b, Kent 1982, Mengoni Goñalons 2008, Mengoni Goñalons y Yacobaccio 2006), las características de la fibra (Reigadas 2000-2, 2008, Wheeler et al. 1995), la morfología ósea (Benavente et al. 1993) y, más recientemente, los isótopos estables (Izeta et al. 2009a, 2010, López Campeny et al. 2005, Mengoni Goñalons 2007, Samec 2011). Precisamente, en este trabajo se pretende contribuir a esta problemática, mediante el entrecruzamiento de distintas líneas de análisis aplicadas sobre los restos de camélidos procedentes de sitios arqueológicos del Valle de Ambato, ocupados entre los siglos VI y X d.C.

\section{El caso de estudio}

El Valle de Ambato se localiza en el sector central de la Provincia de Catamarca, República Argentina, y forma

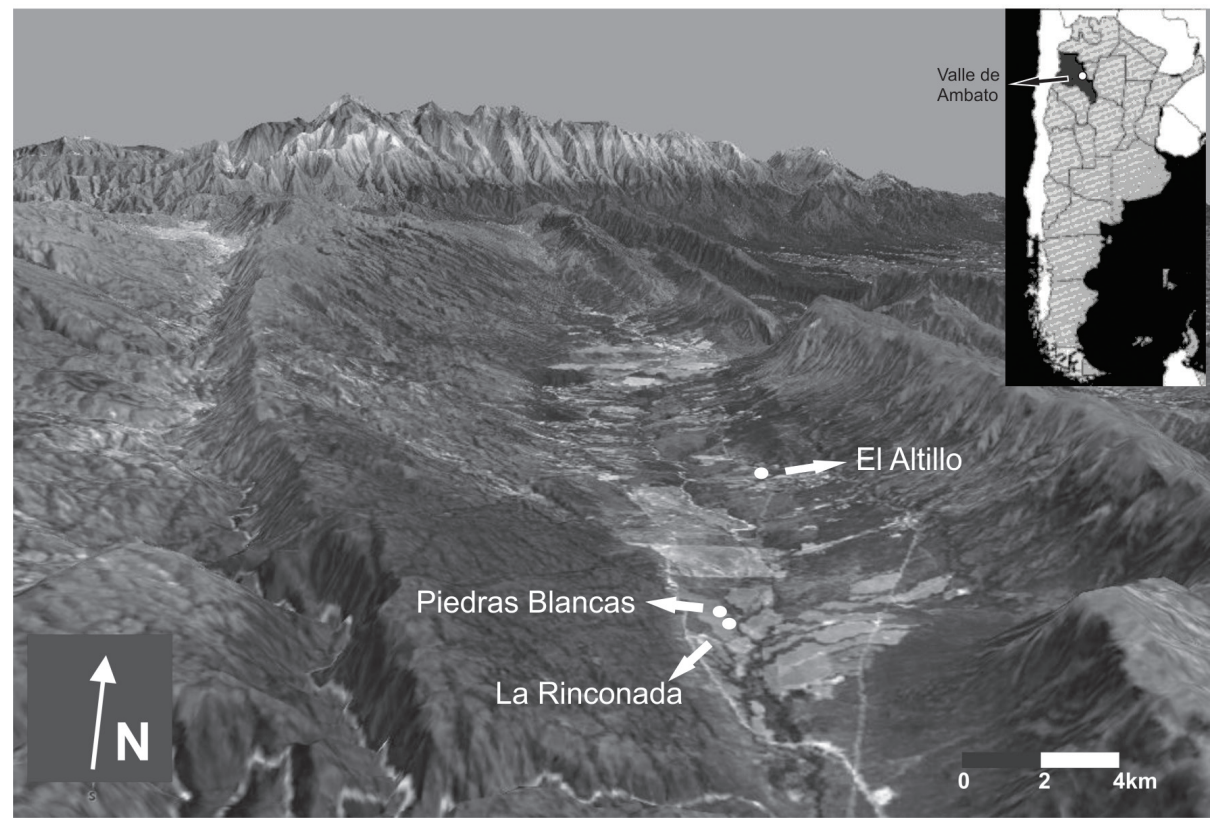

parte de la provincia geológica de las Sierras Pampeanas Noroccidentales. Se encuentra delimitado por el cordón montañoso Ambato-Manchao (4.050 msm) al oeste y por la sierra de Graciana-Balcozna (1.850 msm) hacia el este. Al sur limita con el Valle de Catamarca y al norte con los Altos de Singuil. Sus alturas sobre el nivel del mar varían entre $1.040 \mathrm{~m}$, en las inmediaciones de su límite sur, y $2.278 \mathrm{~m}$, en su ángulo noroeste, en la cima de la Sierra de Humaya. Biogeográficamente, esta región corresponde al Distrito Chaqueño Serrano de la Provincia Chaqueña (Dominio Chaqueño), Región Neotropical (Cabrera 1976, Morláns 2007) (Figura 1).

Entre los siglos VI y XI d.C. en este valle se desarrolló una sociedad heterogénea e internamente diferenciada, caracterizada arqueológicamente como cultura Aguada de Ambato (sensu González, 1998), la cual presentaba un modo de vida basado en una intensificación de la economía y la acumulación de excedentes, un incremento marcado de la población, diversificación de roles sociales, especialización artesanal y desigualdades sociales y políticas institucionalizadas (Laguens 2004). A partir de su concreción, esta sociedad llegó a interactuar con poblaciones en zonas aledañas, que pasaron a integrarse en una misma esfera supra-regional, cada una con características propias (Laguens 2006, Pérez Gollán 1991). Durante esta época, los sitios residenciales se encontraban agrupados en al menos tres conjuntos a la manera de aldeas, con una estructuración y jerarquización similar del espacio. En cada una de estas agrupaciones se destaca la presencia de un sitio principal muy grande, caracterizado por un montículo escalonado, una plaza central y recintos circundantes, rodeado, en las cercanías, por algunos sitios residenciales de múltiples recintos y patios. En la periferia de estos conjuntos, se distribuyen sitios más pequeños y mucho más abundantes que los anteriores (Assandri 2007, Laguens y Bonnin 2005).

Con anterioridad a este período, el valle se encontraba poblado por sociedades distribuidas en asentamientos dispersos, con escasa diferenciación social, un acceso generalizado y una distribución equilibrada de los recursos materiales y sociales, y que presentaban características comunes con zonas vecinas. Estos grupos pueden ser asignados a lo

Figura 1: Mapa del Valle de Ambato con ubicación de sitios.

Figure 1: Ambato Valley map with location of the sites. 
que se ha denominado Periodo Formativo en la literatura arqueológica de las últimas décadas (Laguens 2004, 2006).

Los trabajos arqueológicos realizados en el Valle de Ambato han permitido observar que durante momentos Aguada en los sitios residenciales ubicados en la zona deprimida del mismo, predominó el consumo de camélidos sobre otro tipo de fuente de proteína animal (Dantas 2012). En los piedemontes y faldeos se desarrolló un espacio especializado de producción agropastoril, evidenciado principalmente por la presencia de corrales, terrazas, represas, canales y viviendas (Figueroa 2012, Figueroa et al. 2010). Asimismo, se hallaron restos de camélidos formando parte de contextos ceremoniales o rituales, en sitios de elite y ceremoniales exclusivamente, lo que permite pensar que estos animales pudieron tener un alto valor simbólico y que habrían sido utilizados como bienes de prestigio y capital social (Laguens 2004, 2007). Por consiguiente, la recurrente presencia de los camélidos llevó a que consideremos la necesidad de la realización de estudios que permitan diferenciarlos interespecíficamente, lo que posibilitaría distinguir no solo entre animales domésticos y silvestres, sino también profundizar el conocimiento sobre las estrategias de adquisición y manejo de los mismos.

\section{Materiales y Métodos}

El material analizado proviene del sitio Piedras Blancas que se ubica en el sector deprimido del valle, a una altura de 1.040 metros sobre el nivel del mar. Es un sitio que presenta un montículo y un sector con construcciones, el cual fue definido como una residencia de elite de grandes dimensiones (Assandri, 2007). En base a los fechados radiocarbónicos se puede situar su período de ocupación entre el 600 y 1000 d.C. (1370 \pm 70 A.P., $1340 \pm 40$ A.P., $1230 \pm 80$ A.P., $1040 \pm 50$ A.P., $1000 \pm$ 70, $920 \pm 70$ A.P.) (Laguens 2006, Marconetto 2008). Los materiales óseos recuperados en los distintos sectores de Piedras Blancas suman 5.895 especímenes, de los cuales $2.739(46,5 \%)$ fueron identificados anatómicamente y taxonómicamente y 3.156 (53,5\%) fueron clasificados como no-identificables. Dentro de los mismos, los huesos de Camelidae son predominantes, representando un $78 \%$ de los especímenes identificados a nivel de familia (Dantas 2012).

Con el propósito de cumplimentar los objetivos planteados anteriormente, en este trabajo se realizaron dos tipos de análisis a los restos identificados como Camelidae: estudios osteométricos y de isótopos estables.

La implementación del método osteométrico se realizó siguiendo la metodología planteada por Menegaz et al. (1988, 1989), resumida y utilizada por Cardich e Izeta (1999-2000) e Izeta (2004, 2007a). De este modo, para cada una de las medidas registradas se realizaron análisis cuantitativos multivariados -Análisis de Conglomerados (UPGMA, Unweighted Pair Group using Arithmetical Averages) y Análisis de Componentes Principales (ACP)-. Estos análisis se realizaron utilizando los softwares MultiVatiate Statistical Package versión 3.13c y PAST versión 1.94b. Para la medición de los huesos del esqueleto postcraneal se emplearon los caracteres propuestos por Izeta (2004, 2007b), Kent (1982) y von den Driesch (1976). Únicamente se consideraron aquellos especímenes que se encontraban fusionados, que no presentasen una termoalteración superior al estadio de quemado y que tuvieran los puntos de medición necesarios (Elkin 1996, von den Driesch 1976). Las falanges proximales fueron separadas en delanteras y traseras siguiendo la caracterización morfológica realizada por Kent (1982), segregación que permite discriminar de un modo más efectivo a los elementos según su asignación por especie (Cartajena Fasting 2009, Izeta 2007a, Izeta et al. 2009d, Kent 1982).

Los datos métricos de los taxones de referencia fueron obtenidos de diferentes fuentes (Belotti López de Medina 2007, Izeta 2004, Izeta et al. 2009b, Mengoni Goñalons com. pers. 2008, Srur e Izeta 2008, Yacobaccio et al. 1997/1998). En el caso del guanaco se tuvo la precaución de que procediera del noroeste argentino, siguiendo lo señalado por varios autores (Cartajena y Concha 1997, Izeta et al. 2009b, Mengoni Goñalons 2008, Mengoni Goñalons y Yacobaccio 2006, Olivera y Elkin 1994), para evitar los sesgos producidos por la variación geográfica en el tamaño de este taxón.

En cuanto a los análisis de isótopos estables, se realizaron estudios de $\delta^{13} \mathrm{C}$ a elementos del esqueleto apendicular de camélidos, que se encontraban en buen estado de conservación (es decir, que no se encontraban quemados, meteorizados, con depositación química, etc.). Los análisis fueron realizados en el Instituto de Geocronología y Geología Isotópica-CONICET (INGEIS) y los resultados de los mismos se encuentran publicados en Izeta et al. 2009a y 2010 (ver estos trabajos para mayor detalle sobre la metodología empleada para su procesamiento).

\section{Resultados y Discusión}

Se efectuaron estudios osteométricos sobre 35 especímenes, que incluyen falanges proximales y mediales, metapodios, cuneiformes, radioulnas, escápulas, tibias y fémures (Tabla 1). En las Figuras 2 y 3, se presentan los resultados del análisis realizado a los metapodios distales, donde se puede observar en primer lugar como los elementos de vicuña se separan claramente de los camélidos de mayor tamaño y de los especímenes arqueológicos. En segundo lugar, se puede distinguir que, dentro del grupo de camélidos grandes, algunos especímenes de Piedras Blancas se asocian con las llamas y otros con los guanacos de referencia. Las variables consideradas son MCARP77 o Bd, MCARP78, MCARP79, 


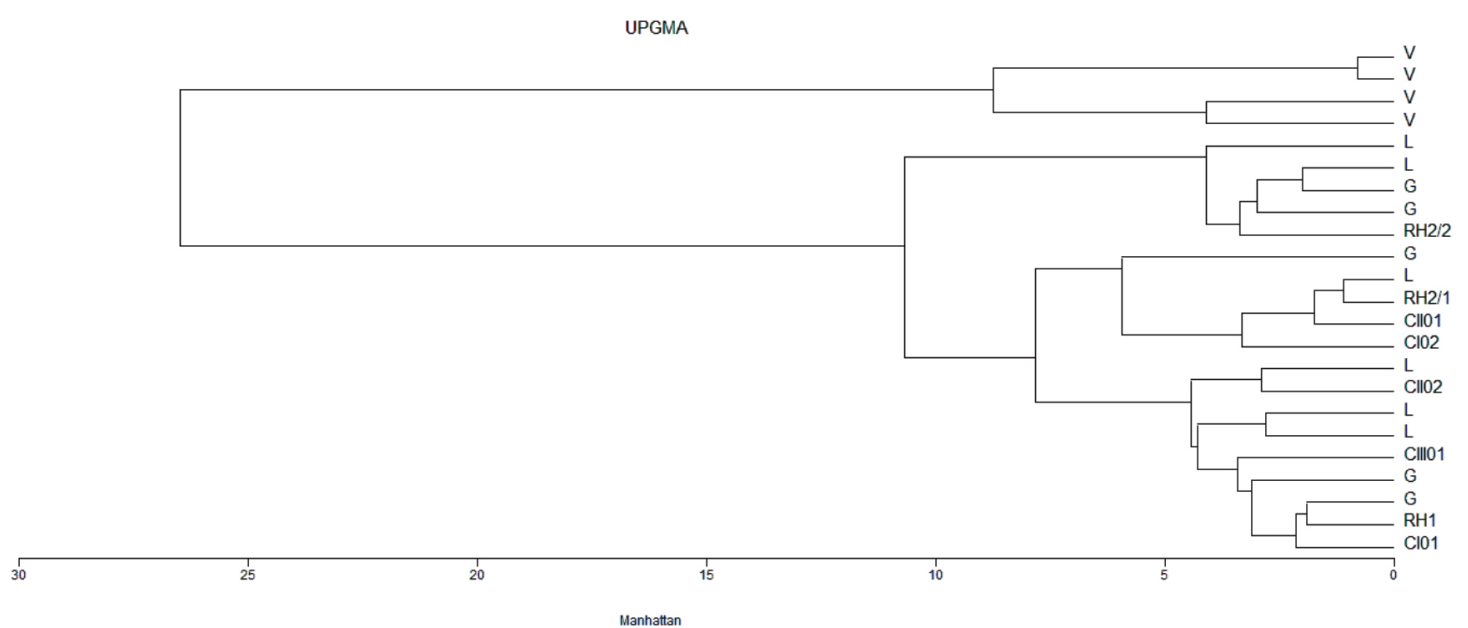

Figura 2: Resultado del Análisis de Conglomerados del metapodio distal. V=Vicugna vicugna de referencia; G= Lama guanicoe de referencia; L= Lama glama de referencia; el resto corresponde a elementos arqueológicos de Piedras Blancas.

Figure 2: Results of the Cluster Analysis of distal metapodia. V= reference Vicugna vicugna; G= reference Lama guanicoe; L= reference Lama glama; the remainder are archaeological elements of Piedras Blancas.

MCARP80, MCARP81 de Kent (1982) y von den Driesch (1976).

De este modo, los resultados alcanzados permitieron discriminar tres agrupamientos, uno que se asocia con las vicuñas, otro con las llamas y un tercero con los guanacos de referencia (Tabla 1). En este último caso, debido a que, como ya fue señalado por varios investigadores (Mengoni Goñalons 2008, Yacobaccio 2010, entre otros), el rango de tamaño del guanaco Andino se superpone con el de las llamas más pequeñas, se optó por asignar estos especímenes a la categoría de "Ilama-guanaco", siguiendo a López (2003) y Olivera y Grant (2009), entre otros.
La presencia de este grupo "Ilama-guanaco" planteó la dificultad de distinguir dentro del mismo entre llamas pequeñas y guanacos, con las consiguientes diferencias en las estrategias de aprovisionamiento que ambos implican (pastoreo versus caza). Cabe mencionar que, en la actualidad, en el Valle de Ambato o sus proximidades no hay poblaciones de guanacos y tampoco se sabe fehacientemente que haya habido en el pasado. A lo que se suma, que en esta categoría se incluyen el $45,7 \%$ de los especímenes analizados (Tabla 1).

Respecto a los análisis de isótopos estables de $\delta^{13} \mathrm{C}$, se realizaron este tipo de estudios sobre una muestra de cuatro (25\%) especímenes procedentes de Piedras

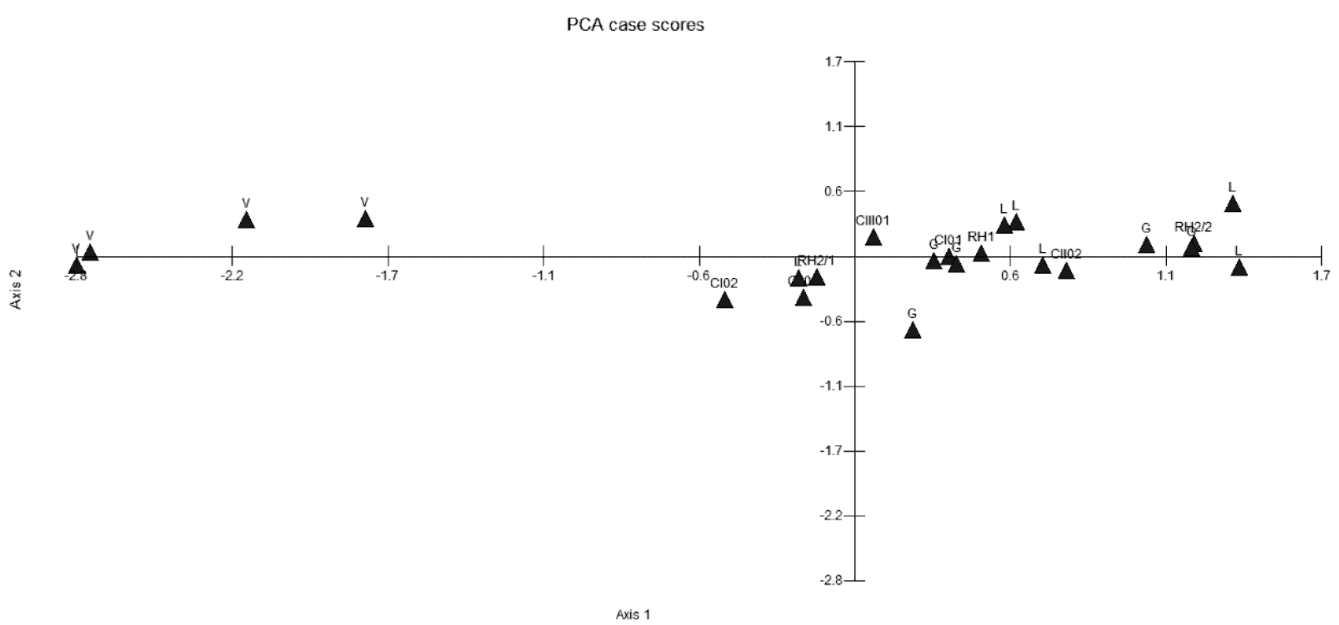

Figura 3: Resultado del Análisis de Componentes Principales del metapodio distal. V=Vicugna vicugna de referencia; G= Lama guanicoe de referencia; L= Lama glama de referencia; el resto corresponde a elementos arqueológicos de Piedras Blancas.

Figure 3: Results of the Principal Component Analysis of distal metapodia. V= reference Vicugna vicugna; $G=$ reference Lama guanicoe; L= reference Lama glama; the remainder are archaeological elements of Piedras Blancas. 


\begin{tabular}{lccc}
\hline Elemento & $\begin{array}{c}\text { Vicugna } \\
\text { vicugna }\end{array}$ & Lama glama & $\begin{array}{c}\text { Llama- } \\
\text { guanaco }\end{array}$ \\
\hline Falange 1 & 3 & 4 & 4 \\
Falange 2 & - & 6 & - \\
Metapodio & - & 4 & 4 \\
Cuneiforme & - & 1 & - \\
Tibia & - & - & 2 \\
Fémur & - & - & 5 \\
Radioulna & - & - & 1 \\
Escápula & - & 1 & - \\
Total & $\mathbf{3 ( 8 , 6 \% )}$ & $\mathbf{1 6}(\mathbf{4 5 , 7 \% )}$ & $\mathbf{1 6}(\mathbf{4 5 , 7 \% )}$ \\
\hline
\end{tabular}

Tabla 1: Resultados de los análisis osteométricos.

Table 1: Osteometric analysis results.

Blancas, correspondientes a elementos del esqueleto apendicular, incluyendo metapodios, tibias y fémures, y que habían sido adscriptas a la categoría "llamaguanaco", mediante los estudios osteométricos. Los valores de $\delta^{13} \mathrm{C}$ obtenidos oscilan entre $-9,9 \%$ y $-13,1 \%$. (Tabla 2: muestras PB00 RH N104 41, PB99 C56 N3 300, PB00 RH N106 17, PB99 C15 44).

Estos análisis se efectuaron en forma conjunta a otras 11 muestras provenientes del mismo sitio, que habían sido asignadas a la categoría llamas, a los que se sumó una muestra de camélido indeterminado realizada con anterioridad (Izeta et al. 2009a). Estos estudios dieron como resultado valores que varían entre $-9,5 \%$ y $-12,7 \%$. Para poder interpretar estos resultados, Izeta y colaboradores $(2009 a, 2010)$ utilizaron como marco de referencia los valores de isótopos de $\delta^{13} \mathrm{C}$ realizados en llamas, guanacos y vicuñas modernas ${ }^{1}$ (Fernández y Panarello 1994, 1999-2001a, 1999-2001b, Fernández et al. 1991, Panarello y Fernández 2002, Schoeninger y DeNiro 1983). Asimismo, en lo que respecta a la ecología isotópica del Valle de Ambato, estos autores observaron que en los distintos pisos vegetales (i.e. bosque serrano, pastizal-arbustal y pastizal de altura) las especies vegetales con capacidad forrajera disponibles registran patrones fotosintéticos $C_{3}$ y $C_{4}$ las cuales se distribuyen indistintamente en los mismos, sin presentar algún tipo

\footnotetext{
${ }^{1}$ En estos trabajos, los camélidos domésticos que pastorean en ambientes por debajo de los $3.900 \mathrm{msnm}$ presentan los valores de $\delta^{13} \mathrm{C}$ más enriquecidos (por encima de $-14,5 \%$ ). Los guanacos y vicuñas, en ambientes por encima de los $3.900 \mathrm{msnm}$, presentan valores que van de $-19,0 \%$ a $-20,4 \%$. Para zonas más bajas, las vicuñas muestran resultados que van de $-14,6 \%$ a $-18,7 \%$, promediando $-16,4 \%$ o (Fernández y Panarello 1999-2001b; Samec 2011). Por su parte, Tykot (2006) menciona que, a nivel general, los animales que tienen una dieta centrada exclusivamente en plantas $C_{3}$ la transferencia al colágeno de $\delta^{13} \mathrm{C}$ es de aproximadamente $-21,5 \%$, en tanto que, en el otro extremo, aquellos que se alimentan exclusivamente con plantas $C_{4}$ presentan valores de $-7,5 \%$. De esta manera, una dieta constituida por una mezcla de $50 \%$ de plantas $C_{3}$ y $50 \%$ de $C_{4}$ produce un valor de $\delta^{13} \mathrm{C}$ de @ $-14,5 \%$.
}

de correlación entre la altitud y los parches de vegetación (Izeta et al. 2009a).

De esta forma, estos resultados muestran que en Piedras Blancas, estos valores enriquecidos de $\delta^{13} \mathrm{C}$ representan una ingesta elevada de plantas $C_{4}$, aproximándose a los valores presentados para maíces sur-andinos y distanciándose, a su vez, de los valores registrados para los recursos herbáceos locales (Izeta et al. 2009a, 2010). Por lo tanto, estos datos apuntan a una especialización en el modo de alimentación de los animales mediante el consumo de maíz en una elevada proporción, a partir de los restos agrícolas (Figueroa et al. 2010, Izeta et al. 2009a, 2010).

Además, se realizaron este mismo tipo de estudios a materiales procedentes del sitio El Altillo que presenta una ocupación previa a momentos Aguada (Tabla 2, Figura 4). En este caso los valores obtenidos son más variables (entre $-11,8 \%$ y $-17,1 \% 0$ ), lo que muestra que los camélidos del sitio más temprano estaban alimentándose con plantas con patrones fotosintéticos $C_{3}$ y $C_{4}$. Esto se asemeja al modo en que se distribuyen las especies vegetales en los distintos pisos del valle. Por lo tanto, estos resultados apuntan a una alimentación de los animales basada en el pastoreo a campo abierto (Figueroa et al. 2010, Izeta et al. 2009a, 2010).

Entonces, estos análisis nos están mostrando que en el Valle de Ambato para momentos Aguada los camélidos que se agrupaban dentro del grupo de animales de tamaño grande se estaban alimentando con altas proporciones de restos agrícolas de maíz .

A partir de estos datos consideramos la posibilidad de que los estudios de isótopos de carbono realizados a los especímenes que se agrupan dentro de la categoría "Ilama-guanaco" nos posibilitarán distinguir dentro de este grupo entre llamas pequeñas y guanacos. De este modo, en el caso de ser animales silvestres (guanacos), se esperaba que de ser locales tendrían que presentar una alimentación que combina plantas $C_{3}$ y $C_{4^{\prime}}$ como se observó con las muestras procedentes de El Altillo, y en caso de proceder de la Puna catamarqueña o la Sierra del Aconquija, donde se encuentran actualmente, los valores tendrían que ser más negativos debido a una dieta con mayores proporciones de plantas $\mathrm{C}_{3}$ (Fernández y Panarello 1999-2001b, Panarello y Fernández 2002, Samec 2011). De haberse obtenido valores similares a los señalados, sin embargo, tampoco sería posible aseverar que se trató de guanacos, ya que los restos de llamas procedentes de la misma región o que recibieron una alimentación con la misma proporción de plantas $C_{3}$ y $C_{4}$ arrojarían valores semejantes.

\footnotetext{
2 El manejo de los rebaños y su integración en un sistema de producción agropastoril fue desarrollado más extensamente en Dantas (2010), Figueroa et al. (2010) e lzeta et al. (2009a, 2010).
} 


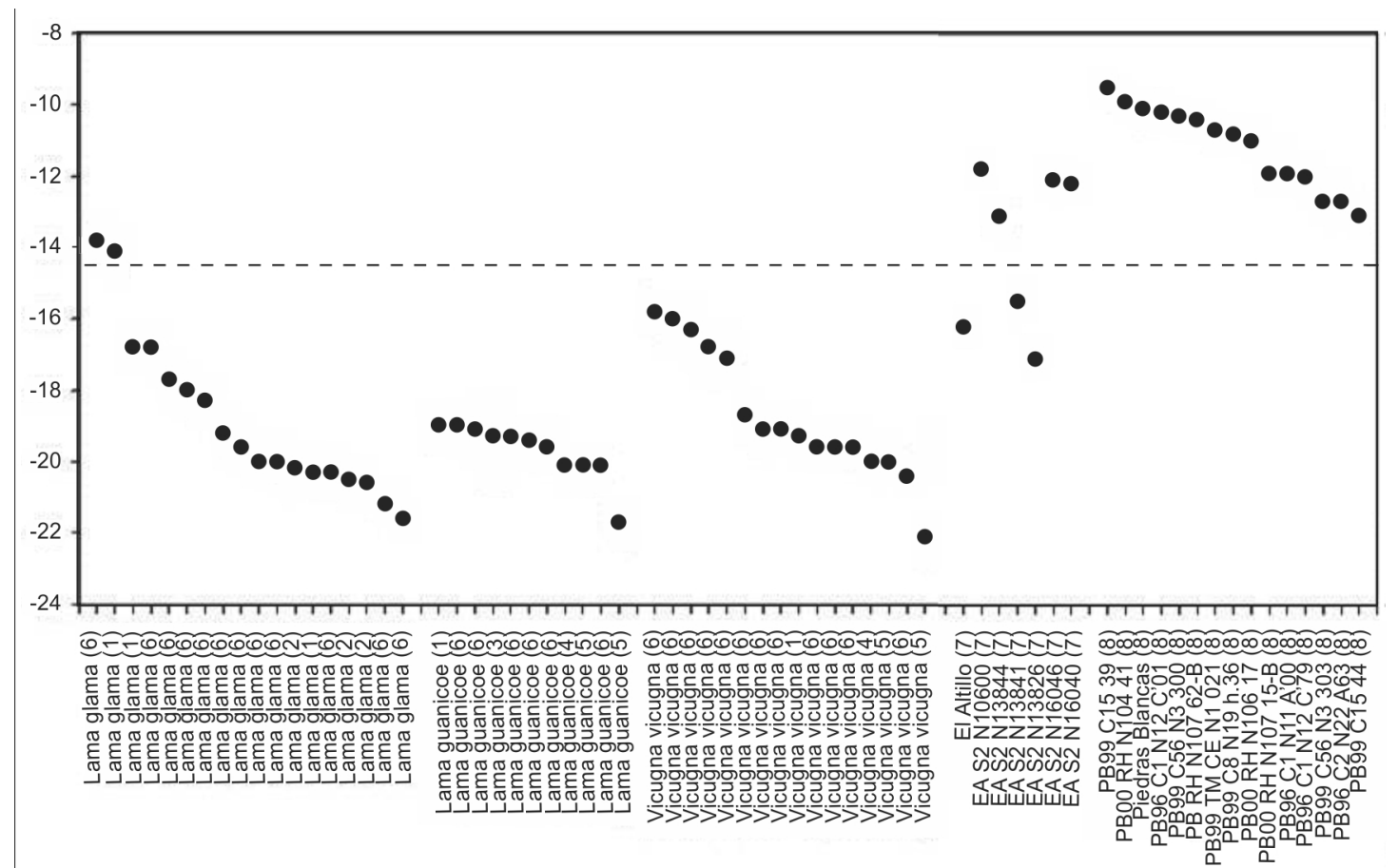

Figura 4: Resultados de los análisis de isótopos de carbono (modificado de Izeta et al. 2010). Referencias: (1) datos de Fernández et al. (1991); (2) datos de Schoeninger y DeNiro (1983); (3) datos de Fernández y Panarello (1994); (4) datos de Panarello y Fernández (2002); (5) datos de Fernández y Panarello (1999-2001a); (6) datos de Fernández y Panarello, (1999-2001b); (7) datos de El Altillo; (8) datos de Piedras Blancas.

Figure 4: Carbon isotope analysis results (modified from lzeta et al. 2010). References: (1) data from Fernández et al. (1991); (2) data from Schoeninger and DeNiro (1983); (3) data from Fernández and Panarello (1994); (4) data from Panarello and Fernández (2002); (5) data from Fernández and Panarello (1999-2001a); (6) data from Fernández and Panarello, (1999-2001b); (7) data from El Altillo; (8) data from Piedras Blancas.

De todas formas, los valores obtenidos se alejan de ambas posibilidades y se asemejan a los registrados para los especímenes de Piedras Blancas determinados osteométricamente como llamas, inclusive uno de ellos presenta uno de los resultados más positivos de la muestra $(-9,9 \%$ ). Uno de los restos óseos (PB99 C15 44) exhibe valores levemente más bajos, pero que no difieren demasiado de los observados para los otros especímenes del mismo sitio (Tabla 2 y Figura 4).

En consecuencia, se puede observar que la mayor parte de los animales agrupados dentro del grupo "Ilama-guanaco" se habrían alimentado con un rango restringido de vegetales, con un alto componente de plantas $\mathrm{C}_{4}$, asemejándose a lo observado para el grupo llamas, indicando una alimentación controlada de los mismos. Esto lleva a considerar que se trató de animales domésticos de menor porte, es decir, llamas pequeñas. No obstante, la presencia de un ejemplar con valores levemente más negativos hace necesario que no se descarte completamente la presencia, en bajos porcentajes, de guanacos.

Resultados similares fueron alcanzados en los estudios realizados en La Rinconada, un sitio ceremonial ubicado en las proximidades de Piedras Blancas, por Svoboda (2010) y Svoboda y Eguia (2010), a través de análisis osteométricos y de isótopos estables. Estas autoras observaron, mediante la medición de falanges proximales, la presencia de llamas pequeñas o guanacos. Estos datos sumados a los valores de isótopos de $\delta^{13} \mathrm{C}$ obtenidos $(-11,2 \%$, $-11,8 \%$ y $-13,1 \% 0)$, las llevó a plantear la posibilidad de que se traten de llamas pequeñas que recibían una alimentación dirigida, con altas proporciones de maíz.

Lo observado en Piedras Blancas y La Rinconada, también se puede vincular con el trabajo de Yacobaccio (2010), quien distingue, a través de la aplicación de la técnica mixture analysis a llamas actuales, una superposición en la medida de estos animales con los guanacos norandinos. Asimismo, obtuvo resultados semejantes en muestras arqueológicas de tres sitios de la Quebrada de Humahuaca (La Huerta, Banda del Perchel y Til. 22), es decir, registró la presencia de diferentes poblaciones de tamaños entre los restos de camélidos de esos sitios, una de las cuales se aproxima a las llamas de mayores dimensiones y otra se traslapa con los guanacos. En base a la semejanza de tamaños entre las llamas actuales y los casos arqueológicos, y a consideraciones contextuales, Yacobaccio (2010) concluye por interpretar la presencia de llamas pequeñas en los sitios arqueológicos.

\section{Conclusiones}

La integración de estudios osteométricos y de isótopos 


\begin{tabular}{|c|c|c|c|c|}
\hline Muestra & Grupo & Elemento & $\delta 13 C(\%)$ & Código de Laboratorio \\
\hline PB99 C15 39 & Llama & Metapodio & $-9,5$ & AIE 21565 \\
\hline PB00 RH N104 41 & Llama/guanaco & Fémur & $-9,9$ & AIE 21564 \\
\hline Piedras Blancas & Lama sp. & Hueso largo & $-10,1$ & AIE 13249 \\
\hline PB96 C1 N12 C’01 & Llama & Metapodio & $-10,2$ & AIE 21555 \\
\hline PB99 C56 N3 300 & Llama/guanaco & Metapodio & $-10,3$ & AIE 21561 \\
\hline PB00 RH N107 62-B & Llama & Metapodio & $-10,4$ & AIE 21557 \\
\hline PB99 TM CE N1 021 & Llama & Metapodio & $-10,7$ & AIE 21554 \\
\hline PB99 C8 N19 h.36 & Llama & Metacarpo & $-10,8$ & AIE 21566 \\
\hline PB00 RH N106 17 & Llama/guanaco & Tibia & $-11,0$ & AIE 21556 \\
\hline PB00 RH N107 15-B & Llama & Metapodio & $-11,9$ & AIE 21553 \\
\hline PB96 C1 N11 A'00 & Llama & Fémur & $-11,9$ & AIE 21559 \\
\hline PB96 C1N12 C’79 & Llama & Húmero & $-12,0$ & AIE 21558 \\
\hline PB99 C56 N3 303 & Llama & Metatarso & $-12,7$ & AIE 21560 \\
\hline PB96 C2 N22 A63 & Llama & Metacarpo & $-12,7$ & AIE 21563 \\
\hline PB99 C15 44 & Llama/guanaco & Fémur & $-13,1$ & AIE 21562 \\
\hline El Altillo & Lama sp. & Metapodio & $-16,2$ & AIE 13248 \\
\hline EA S2 N10600 & Llama & Radioulna & $-11,8$ & AIE 21550 \\
\hline EA S2 N13844 & Llama & Húmero & $-13,1$ & AIE 21549 \\
\hline EA S2 N13841 & Llama & Tibia & $-15,5$ & AIE 21548 \\
\hline EA S2 N13826 & Llama & Unciforme & $-17,1$ & AIE 21547 \\
\hline EA S2 N16046 & Llama & Metacarpo & $-12,1$ & AIE 21551 \\
\hline EA S2 N16040 & Llama & Húmero & $-12,2$ & AIE 21552 \\
\hline
\end{tabular}

Tabla 2: Muestras analizadas de Piedras Blancas y El Altillo.

Table 2: Samples analyzed from Piedras Blancas and El Altillo.

estables posibilitó identificar la presencia durante las ocupaciones Aguada del Valle de Ambato de camélidos domésticos en función de su tamaño relativo y una dieta selectiva concentrada en plantas $C_{4}$. A su vez, dentro de estas poblaciones de animales fue posible distinguir la existencia de dos grupos que podrían responder a variedades de llamas o morfotipos distintos: un grupo de animales de mayor porte, o llamas grandes, y otro de menor porte, o llamas pequeñas, cuyo tamaño se superpone con el rango de tamaños de los guanacos norandinos.

Entonces, en base a los resultados obtenidos, creemos necesario remarcar la potencialidad de la combinación de ambos análisis al momento de abordar la identificación interespecífica de camélidos, particularmente en contextos semejantes al tratado en este artículo, donde este tipo de estudios permitió alcanzar un grado de diferenciación que de otro modo no hubiera sido posible. De este modo, se logró aportar nuevos datos que permiten avanzar en el conocimiento sobre las estrategias de manejo de los animales domésticos dentro del sistema de producción agropastoril que se desarrolló en este valle entre el siglo VI y X d.C.

\section{Agradecimientos}

A los organizadores del simposio "Metodologías y técnicas aplicadas en zooarqueología: avances y perspectivas", por invitarme a publicar el trabajo. A Andrés Laguens, Andrés Izeta, Mariana Mondini y Germán Figueroa por el apoyo y contribuciones al desarrollo de los temas de este trabajo. Este estudio fue financiado por subsidios de FONCyT (PICT $2005 N^{\circ} 34558$ ) y SECYT-UNC.

\section{Bibliografía}

Assandri, S. B. 2007. Procesos de complejización social y organización espacial en el Valle de Ambato, Catamarca, Argentina. Universidad Internacional de Andalucía, Sede Iberoamericana Santa María de la Rábida, La Rábida. En: http://www.unia.es/nuevo_inf_academica/visualizar_file_ Adjunto.asp?ID=3237

Belotti López de Medina, C. 2007. "Zooarqueología del sitio Soria 2 (Depto. de San José, Pcia. de Catamarca) y estudio comparativo del registro zooarqueológico del sur de los valles calchaquíes, para los periodos Formativo y Desarrollos Regionales (Siglos I A.C. y XV D.C.)". Facultad de Filosofía y Letras, Universidad de Buenos Aires, Argentina, 172 páginas. Tesis de Licenciatura.

Benavente Aninat, M. A., L. Adaro Arauena, P. Gecele Ciuffi y C. Cunazza Pailuri. 1993. Contribución a la determinación de especies animales en arqueología: Familia Camelidae y Taruca del Norte. Universidad de Chile, Vicerrectoría Académica y Estudiantil, Departamento Técnico de Investigación, Santiago.

Cabrera, A. 1976. Regiones fitogeográficas Argentinas. Enciclopedia Argentina de Agricultura y Jardinería, vol. 1. ACME, Buenos Aires. 
Cardich, A., A. D. Izeta. 1999-2000. Revisitando Huargo (Perú). Análisis cuantitativos aplicados a restos de Camelidae del Pleistoceno Tardío. Anales de Arqueología y Etnología, 54-55: 29-40.

Cartajena Fasting, I. 2009. Explorando la variabilidad morfométrica del conjunto de camélidos pequeños durante el Arcaico Tardío y el Formativo Temprano en Quebrada Tulán, norte de Chile. Revista del Museo de Antropología, 2: 199-214.

Cartajena, I., I. Concha. 1997. Una contribución a la determinación taxonómica de la familia Camelidae en sitios formativos del Loa Medio. Estudios Atacameños, 14: 71-83.

Dantas, M. 2010. Arqueología de los animales y procesos de diferenciación social en el valle de Ambato, Catamarca, Argentina. Facultad de Filosofía y Humanidades, Universidad Nacional de Córdoba, Argentina, 389 páginas. Tesis Doctoral.

Elkin, D. C. 1996. "Arqueozoología de Quebrada Seca 3: indicadores de subsistencia humana temprana en la Puna Meridional Argentina". Facultad de Filosofía y Letras, Universidad de Buenos Aires, Argentina. Tesis Doctoral.

Fernández, J., V. Markgraf, H. Panarello, M. Albero, F. Angiolini, S. Valencio y M. Arriaga. 1991. Late Pleistocene-early Holocene Environment and climates, fauna, and human occupation in the Argentine Altiplano. Geoarcheology, 6: 251-272.

Fernández, J., H. Panarello. 1994. Estimaciones Paleodietéticas y Ambientales: Esqueletos 1 y 2 Puesto El Rodeo. En: Gradin, C. J., A. M. Aguerre (Eds.), Contribución a la Arqueología del Río Pinturas (Provincia de Santa Cruz), pp. 300-310, Búsqueda de Ayllu, Concepción del Uruguay.

Fernández, J., H. O. Panarello. 1999-2001a. Isótopos estables del carbono en pelo de animales silvestres de ambientes altiplánicos de Argentina. Xama, 12-14: 61-69.

Fernández, J., H. O. Panarello. 1999-2001b. Isótopos del carbono en la dieta de herbívoros y carnívoros de los Andes jujeños. Xama, 12-14: 71-85.

Figueroa, G. G. 2012. Pircas, llamas y maíz. Un estudio arqueológico y etnoarqueológico de sistemas de producción de montaña en el Noroeste Argentino. Editorial Académica Española, Saarbrücken.

Figueroa, G. G., M. Dantas y A. G. Laguens. 2010. Prácticas agropastoriles e innovaciones en la producción de plantas y animales en los Andes del Sur. El Valle de Ambato, Argentina, Primer milenio d.C. International Journal of South American Archaeology, 7: 6-13.
González, A. R. 1998. Cultura La Aguada. Arqueología y diseños. Filmediciones Valero, Buenos Aires.

Izeta, A. D. 2004. "Zooarqueología del Sur de los Valles Calchaquíes: Estudio de conjuntos faunísticos del Período Formativo". Facultad de Ciencias Naturales y Museo, Universidad Nacional de La Plata, Argentina, 430 páginas. Tesis Doctoral.

Izeta, A. D. 2007a. Zooarqueología del sur de los valles Calchaquíes (Provincias de Catamarca y Tucumán, República Argentina): Análisis de conjuntos faunísticos del primer milenio A.D. BAR International Series S1612, Oxford.

Izeta, A. D. 2007b. Interespecific Differentiation of South American Camelids from Archaeofaunal Assemblages in the Southern Calchaquíes Valleys (Argentina). En: Gutiérrez, M., L. Miotti, G. Barrientos, G. Mengoni Goñalons y M. Salemme (Eds.), Taphonomy and Zooarchaeology in Argentina, pp. 47-57. BAR International Series S1601, Oxford.

Izeta, A. D., A. G. Laguens, M. B. Marconetto y M. C. Scattolin. 2009a. Camelid handling in the meridional Andes during the first millennium AD: a preliminary approach using stable isotopes. International Journal of Osteoarchaeology, 19(2): 204-214.

Izeta, A. D., C. Otaola y A. Gasco. 2009b. Estándares métricos y variabilidad en falanges proximales de camélidos sudaméricanos. Su importancia como conjunto comparativo para interpretaciones en arqueología. Revista del Museo de Antropología, 2(1): 169-180.

Izeta, A. D., M. Dantas, M. G. Srur, M. B. Marconetto, A. G. Laguens. 2010. Isótopos estables y manejo alimentario de camélidos durante el primer milenio A.D. en el Valle de Ambato (Noroeste Argentino). En: Bertolino, S., R. Cattáneo y A. Izeta (Eds.), La arqueometría en Argentina y Latinoamérica, pp. 237-242. Editorial de la Facultad de Filosofía y Humanidades, Universidad Nacional de Córdoba, Córdoba.

Kent, J. D. 1982. "The Domestication and exploitation of the South American camelids: methods of analysis and their application to circum-lacustrine archaeological sites in Bolivia and Peru". Washington University, St. Louis. Tesis Doctoral.

Laguens, A. G. 2004. Arqueología de la diferenciación social en el valle de Ambato, Catamarca, Argentina (s. II - VI d.C.): El actualismo como metodología de análisis. Relaciones de la Sociedad Argentina de Antropología, XXIX: 137-161.

Laguens, A. G. 2006. Continuidad y ruptura en procesos 
de diferenciación social en comunidades aldeanas del valle de Ambato, Catamarca, Argentina (s. IV-X d.C.). Chungara, Revista de Arqueología Chilena, 38(2): 211222.

Laguens, A. G. 2007. Contextos materiales de desigualdad social en el valle de Ambato, Catamarca, Argentina, entre los siglos VII y X d.C. Revista Española de Antropología Americana, 37(1): 27-49.

Laguens, A. G., M. Bonnin. 2005. Recursos materiales y desigualdad social en la arqueología de Ambato Catamarca. En La cultura de La Aguada y sus expresiones regionales, pp. 23-33. EUDELAR, La Rioja.

Laker, J., J. Baldo, Y. Arzamendia y H. D. Yacobaccio. 2006. La vicuña en los Andes. En: Vilá, B. (Ed.), Investigación, conservación y manejo de vicuñas, pp. 37-50. Universidad Nacional de Luján, Buenos Aires.

López, G. 2003. Pastoreo y caza de camélidos en el Temprano de la Puna de Salta: Datos osteométricos del sitio Matancillas 2. Intersecciones en Antropología, 4: 17-27.

López Campeny, S. M. L., D. E. Olivera, V. Fernández Varela y J. Peña. 2005. Procesos tafonomicos, subsistencia y uso del espacio: analisis de la arqueofauna de un sitio agropastoril de la Puna Meridional Argentina (Punta de la Peña 9, Antofagasta de la Sierra, Catamarca). Intersecciones en Antropología, 6: 11-28.

Marconetto, M. B. 2008. Recursos forestales y el proceso de diferenciación social en tiempos prehispánicos en el valle de Ambato, Catamarca, Argentina. British Archaeological Reports- South American Archaeology Series, Oxford.

Menegaz, A. N., M. C. Salemme y E. Ortiz Jaureguizar. 1988. Una propuesta de sistematización de los caracteres morfométricos de los matapodios y las falanges de Camelidae. En: Ratto, N. R., A. F. Haber (Eds.), De procesos, contextos y otros huesos, pp. 53-63. Facultad de Filosofía y Letras, U.B.A., Buenos Aires.

Menegaz, A., F. J. Goin y E. Ortiz Jaureguizar. 1989. Análisis morfológico y morfométrico multivariado de los representantes fósiles y vivientes del género Lama (Artiodactyla, Camelidae). Sus implicancias sistemáticas, biogeográficas, ecológicas y biocronólogicas. Ameghiniana. 26(3-4): 153-172.

Mengoni Goñalons, G. L. 2007. Camelid management during Inca times in N. W. Argentina: models and archaeozoological indicators. Anthropozoologica, 42(2): 129-141.

Mengoni Goñalons, G. L. 2008. Camelids in ancient
Andean societies: A review of the zooarchaeological evidence. Quaternary International, 185: 59-68.

Mengoni Goñalons, G. L., H. D. Yacobaccio. 2006. The domestication of South American camelids. A view from the South-Central Andes. En: Zeder, M., D. Bradley, E. Emshwiller y B. Smith (Eds.), Documenting domestication. New genetic and archaeological paradigms, pp. 228244. University of California Press, Berkeley.

Morláns, M. C. 2007. Regiones naturales de Catamarca. Provincias geológicas y provincias fitogeográficas. En: Morláns, M. C. (Ed.), Área Ecología, pp. 1-36. Editorial Científica Universitaria, Catamarca. http://www.editorial. unca.edu.ar/Publicacione \%20on\%20line/Ecologia/index. html (última consulta: 22 de enero de 2010).

Olivera, D. E., D. C. Elkin. 1994. De cazadores y pastores: el proceso de domesticación de camélidos en la Puna Meridional Argentina. Zooarqueología de Camélidos, 1: $95-124$

Olivera, D. E., J. L. Grant, 2009, Puestos de altura de la Puna argentina: zooarqueología de Real Grande 1 y 6 y Alero Tomayoc. Revista del Museo de Antropología, 2:151-168.

Panarello, H. O., J. Fernandez. 2002. Stable isotope carbon isotope measurements on hair from wild animals from altiplanic environments of Jujuy. Radiocarbon, 44: 709-716.

Pérez Gollán, J. A. 1991. La Cultura de la Aguada vista desde el Valle de Ambato. Publicaciones del CIFFYH, Arqueología, 46: 157-174.

Samec, C. T. 2011. "Perspectiva isotópica sobre la alimentación de camélidos domésticos y silvestres de la Puna Jujeña: Construyendo un marco de referencia para estudios arqueológicos". Facultad de Filosofía y Letras, Universidad de Buenos Aires, Argentina. Tesis de Licenciatura.

Schoeninger, M. J., M. J. De Niro. 1983. Nitrogen and Carbon Isotopic Composition of Bone Collagen from Marine and Terrestrial Animals. Geochimica et Cosmochimica Acta, 48: 625-639.

Srur, M. G., A. D. Izeta. 2008. Osteometría de elementos del esqueleto apendicular de Lama glama. Su importancia como conjunto comparativo para estudios arqueológicos. En: Resúmenes XXII Jornadas Argentinas de Mastozoología, pp. 116-117. Villa Giardino, Córdoba.

Svoboda, A. 2010. "Análisis de los conjuntos arqueofaunísticos de un núcleo residencia del La Rinconada (Valle de Ambato, Catamarca)". Facultad de Filosofía y Letras, Universidad de Buenos Aires, Argentina. 
Tesis de Licenciatura.

Svoboda, A., L. Eguia. 2010. Nuevas aproximaciones a los conjuntos arqueofaunísticos del patio (E5) de La Rinconada (valle de Ambato, Catamarca). En: Gutiérrez, M., M. De Nigris, P. Fernández, M. Giardina, A. Gil, A. Izeta, G. Neme y H. Yacobaccio (Eds.), Zooarqueología a principios del siglo XXI. Aportes teóricos, metodológicos y casos de estudio, pp. 593-602. Ediciones Libros del Espinillo, Buenos Aires.

Tykot, R. H. 2006. Isotope Analyses and the Histories of Maize. En: Staller, J. E., R. H. Tykot y B. F. Benz (Eds.), Histories of Maize in Mesoamerica. Multidisciplinary Approaches, pp. 131-142. Academic Press, San Diego. von den Driesch, A. 1976. A Guide to the Measurement of Animal Bones from Archaeological Sites. Bulletin No. 1, Peabody Museum of Archaeology and Ethnology, Cambridge.

Wheeler, J. C. 1982. Aging llamas and alpacas by their teeth. Llama World, I: 12-17.

Wheeler, J. C. 1995. Evolution and present situation of the
South American Camelids. Biological Journal of Linnean Society, 54: 271-295.

Wheeler, J. C. 2006. Historia natural de la vicuña. En: Vilá, B. (Ed.) Investigación conservación y manejo de vicuñas, pp. 25-35. Proyecto MACS Argentina, Buenos Aires.

Wheeler, J. C., A. J. F. Russel y H. Redden. 1995. Llamas and alpacas: preconquest breeds and post-conquest hybrids. Journal of Archaeological Science, 22: 833-840.

Yacobaccio, H. D. 2010. Osteometría de llamas (Lama glama L.) y sus consecuencias arqueológicas. En: Gutiérrez, M., M. De Nigris, P. Fernández, M. Giardina, A. Gil, A. Izeta, G. Neme y H. Yacobaccio (Eds.), Zooarqueología a principios del siglo XXI. Aportes teóricos, metodológicos y casos de estudio, pp. 65-75. Ediciones Libros del Espinillo, Buenos Aires.

Yacobaccio, H. D., C. M. Madero, M. P. Malmierca y M. C. Reigadas. 1997/1998. Caza, domesticación y pastoreo de camélidos en la Puna Argentina. Relaciones de la Sociedad Argentina de Antropología, XXII-XXIII: 389418. 